DIVISION OF THE HUMANITIES AND SOCIAL SCIENCES

CALIFORNIA INSTITUTE OF TECHNOLOGY

PASADENA, CALIFORNIA 91125

CHOICE AND INDIVIDUAL WELFARE

Christopher P. Chambers

California Institute of Technology

Takashi Hayashi

University of Texas, Austin

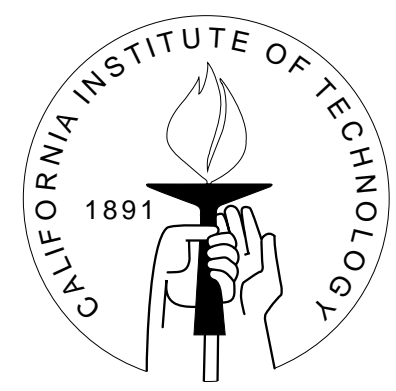

SOCIAL SCIENCE WORKING PAPER 1286

March 2008 


\title{
Choice and individual welfare
}

\author{
Christopher P. Chambers Takashi Hayashi
}

\begin{abstract}
We propose an abstract method of systematically assigning a "rational" ranking of outcomes to choice data which may not be rationalizable in any sense. An individual welfare functional maps stochastic choice functions into weak orders. A stochastic choice function gives the empirical frequency of choices for any possible opportunity set (framing factors may also be incorporated into the model); we call such general choice functions "choice distributions." We require that for any two alternatives $x$ and $y$, if our individual welfare functional recommends $x$ over $y$ given two distinct choice distributions, then it also recommends $x$ over $y$ for any mixture of the two choice distributions. Together with some mild technical requirements, such an individual welfare functional must weight every opportunity set and assign a utility to each alternative $x$ which is the sum across all opportunity sets of the weighted probability of $x$ being chosen from the set. It therefore requires us to have a "prior view" about how important a choice of $x$ from a given opportunity set is.
\end{abstract}

JEL classification numbers: D60

Key words: behavioral economics, welfare economics, axiomatic approach, choice theory 


\title{
Choice and individual welfare *
}

\author{
Christopher P. Chambers Takashi Hayashi
}

\section{Introduction}

\subsection{Behavioral welfare debates}

Standard welfare economics is grounded on the assumption that each individual has a single and consistent preference ranking over social outcomes or her own consumption. ${ }^{1}$ In such a context, leaving aside the problem of strategic misrepresentation of preference, what is better or worse for a given consumer is obvious. - Ask him, or just let him choose.

The assumption that individuals rank outcomes consistently has long been challenged by the community of experimental psychologists. They find various types of behavioral anomalies and biases. ${ }^{2}$ Recently, the community of economists has seriously addressed such challenges; and the research program of "behavioral economics" (or psychology and economics) has become established as an accepted framework of descriptive analysis.

This development, however, brings up a new issue. How should welfare analysis be conducted when behavior may be inconsistent with the maximization of some preference? When choices are inconsistent, the concepts of "better" or "worse" become meaningless for individuals (let alone for groups). Let us illustrate this through the following example.

Example 1 You are going to give a gift to your friend. You know that they make the following choices: they choose $x$ out of set $\{x, y\}$, and they choose $y$ out of set $\{x, y, z\}$. Which one of $x, y$ and $z$ should you give them?

Two natural arguments could be put forth. Perhaps $y$ should be given, as it is chosen from a larger set. On the other hand, perhaps $x$ is better, as it beats $y$ in a direct comparison. Within the standard framework of revealed preference, we can say nothing.

*We would like to thank Colin Camerer, Jeff Ely, and Matt Rabin for helpful discussions and comments. All errors are our own.

${ }^{1}$ In general, such a preference need not be complete or transitive.

${ }^{2}$ Since there is a huge literature about it, here we just cite some books, Kahneman, Slovic and Tversky [14], Kahneman and Tversky [15], and Thaler [29]. 
Which view should be taken, if either, cannot be answered with the tools of classical economics. Any ranking of the two outcomes amounts to an outside value judgment. In any case, no theoretical examination has been undertaken studying how we can weight different choice situations.

Recently, Thaler and Sunstein [30,31] have proposed what they call "libertarian paternalism," which has been quite controversial (see for example Mitchell [22]). It (very) roughly states that choice should be respected whenever it is consistent, but intervention by an authority becomes necessary when individuals exhibit choice inconsistency. When individuals exhibit inconsistency, something must be done, and in this sense paternalism is somehow necessary. They do not provide any investigation as to whether the intersection of 'libertarianism' and 'paternalism' is nonempty, or to understand rules meeting both of these conditions. Our approach is to work in an abstract environment, making systematic recommendations for a paternalistic social planner.

Thaler and Sunstein can be criticized by saying that "irrational behavior" is simply an artifact of misspecification of the choice setting. It is quite often the case that certain actions appear to be 'irrational' or 'paradoxical' simply because the observer fails to account for all the relevant factors. For example, there are many economic instances in which an action is irrational in the short-run but rational in the long-run. In such cases, seemingly irrational or paradoxical behavior can be rationalized by including the missing factors or by suitably redefining the choice domain.

We make no judgment here about whether all behavior can be rationalized in such manner. However, we need to be prepared for the case that choices are not rationalizable even when all the relevant factors are taken into account.

There is another issue: Even if choice is rationalizable by means of a single consistent preference ranking, it may not immediately equate to the notion of welfare. A number of authors claim that economists should make a distinction between 'decision utility' that governs choice, and 'experienced utility' that determines subjective level of welfare (see for example Kahneman, Wakker and Sarin [16]), although retrospective evaluation of outcomes brings up a methodological problem such as rationalization.

Also, a number of studies in neighboring disciplines such as neuroscience claim that choices have rather little to do with subjective perception of welfare, and that it is determined by (or at least related to) the amount of certain chemicals in brain [4].

Thus, many behavioral economists would argue that individuals systematically make many mistakes and hence choice and welfare may not be related. Economic problems consist of choice data, and thus economists have traditionally used choice as a proxy for individual welfare. To some extent, the issue is tautological. Welfare is whatever we define it to be, and economists usually define welfare through choice. Our approach is 
similar in spirit to the revealed preference paradigm. Given observed choice data, what preference is reasonable to use as a proxy for individual welfare?

\subsection{Our approach}

In this paper, we consider choice data as the only data available to an economist that can be used to determine individual welfare. We do not deny that observations other than choice, such as retrospective evaluation and brain activities, may be important and useful in evaluating welfare. Instead, we try to understand what we can say using choice data alone. Our approach does not a priori preclude the possibility of using data other than choice.

We take an axiomatic approach, so as to systematically characterize the economist's possibilities. We imagine that the economist should follow a certain rule in providing welfare prescriptions for inconsistent choice data. Such a rule is modeled as a function mapping arbitrary choice data into a ranking over alternatives. The data is taken to be a list of empirical distributions of choices indexed by choice situations. In general, these empirical distributions may or may not be rationalized either in the deterministic or stochastic sense. Let us call such a rule an individual welfare functional.

We interpret this individual welfare functional as a "suitable" proxy for individual welfare; however we do not necessarily mean to imply that the assigned preference is a "true" or "correct" preference (it is not even clear that a true preference exists for an individual). However, if individual choice is rationalizable in the standard revealed preference framework, given that we only observe choice data, we believe it is natural that the assigned ranking should coincide with the rationalizing preference.

This paper is not concerned with why choice may be irrational. Common arguments are that there is some true, underlying, rational preference and that individuals merely make mistakes, or that there are multiple selves and individuals aggregate these selves according to some rule. If we know these statements to be true, then the underlying problem is one of inference. However, discussions of "true preference" and mistakes are tautological when choice is the only available data. We cannot distinguish an individual who makes mistakes from an individual who simply has no consistent preference. Even if it were known the individual was making mistakes, the nature of the mistakes would have to be understood before being able to identify true preference, necessarily requiring a degree of faith in the economist. With this in mind, we take an agnostic position on this issue and view our individual welfare functional as a normative prescription.

We now discuss our axioms. Our basic axiom is called Combination. It is a consistency requirement in aggregating situation dependent choices. It roughly says, if two choice

situations both support $x$ over $y$, then the choice data obtained by joining the two also supports $x$ over $y$. 
Our main result is as follows. The combination axiom, combined with other technical axioms, implies a form of linear aggregation. The ranking of alternatives is determined by a utility function. The utility of any alternative in our representation is merely a linear function of the distributions. In general, the utility of $x$ can depend on the probability that some alternative $y$ is chosen, where $y$ may not even be present in any situation where $x$ is present.

While the general family of individual welfare functionals may be interesting for many environments, oftentimes it may be unnatural to allow the utility of an alternative to depend on the probability that unrelated alternatives are chosen. To remedy this situation, we impose an additional axiom, binary independence, specifying that the ranking between two alternatives should not be affected by empirical choice probabilities of other alternatives. A related axiom, binary monotonicity, specifies that utility of an alternative should respond positively to the probability that it is chosen. The additional axioms deliver a more structured class of individual welfare functionals. In this class, every pair consisting of a situation and a choice from that situation has its own weight. This weight specifies a prior view about how important it is to choose that alternative in that situation. The utility of the alternative is then just the sum across all situations of the corresponding weight times the probability the alternative was chosen in that situation.

The requirement that the individual welfare assignment should respect choice narrows down the possible range of prior weights. That is, the role of the weights is only in balancing inconsistent choices. They have no implication when individual choice is indeed consistent. Thus, while our individual welfare functional is necessarily paternalistic when choice data is inconsistent, it is also libertarian whenever possible.

\subsection{Related literature}

In a recent paper, Green and Hojman [9] consider an individual as a 'society' that is populated with multiple personalities who may have conflicting preferences. They apply a scoring rule as the method of aggregating the conflicting preferences, determining a choice function. The scoring rule approach is then used to make statements about individual welfare, assuming cardinal information on the utilities of the multiple selves. Thus, they must first discuss the question as to which functions can be rationalized by this type of "multiple selves" model. They find that the model has little in the way of testable implications; and further, that it is not in general identified. Several lists of multiple selves may induce the same choice functions. Hence, only bounds on welfare statements are available in this model. Our work may be viewed as a complement to theirs. However, we consider a general situation where the source of possible choice inconsistency may be of any kind-as opposed to inconsistency which can be rationalized by the multiple selves approach.

Bernheim and Rangel $[2,3]$ also discuss welfare when choice may not be rationalizable. 
Their approach is to define several binary relations through choice. They also consider different framing factors, and essentially define an individual to be unambiguously better off with $x$ over $y$ if she ever chooses $x$ when $y$ is available and always chooses $x$ if $y$ is chosen. Such relations can be highly incomplete and often provide no deterministic prescription for ranking a given pair of alternatives. In this sense, the approach of Bernheim and Rangel does not allow us to conclude when an individual is "better off" except in very limited circumstances. Except for very basic principles such as Pareto optimality, much of welfare economics is grounded on principles which requiring alternatives to be compared. Our work complements the Bernheim-Rangel approach by providing resolute prescriptions for such comparisons.

There is a huge literature on revealed preference theory characterizing conditions under which choice can be rationalized by maximization of preference. The classic papers are by Samuelson $[26,27]$, who first provided the rationalizability condition in the market setting. For abstract choice functions, Chernoff [6], Arrow [1] and Sen [28] provide the conditions in the deterministic setting under which observed choice is rationalizable. In the setting of stochastic choice function, Luce [20] provides the conditions under which the choice probability of an alternative is proportional to some cardinal utility assigned to it. Falmagne [7] provides the conditions under which there is a probability measure over preferences such that the choice probability of an alternative is given by the probability of preferences in which it is maximal.

Recent papers consider rationazability in more general senses. Kalai, Rubinstein and Spiegler [17] provide a condition under which observed choice is rationalizable as a maximal element according to multiple criteria, in which choices need not come from a single objective. Tyson [32] provides a condition under which observed choice is explained as if it is obtained by satisficing an underlying preference, which is a milder procedural rationality requirement than maximization. Masatlioglu and Ok [23] consider an extended domain of choice functions in which a choice problem comes in the form of a pair of a set and a reference point. They provide a condition under which observed choice is rationalizable by maximizing preference with a bias toward the reference point. Rubinstein and Salant [24] consider the setting that an individual makes choice from a list, in which the order of appearances of alternatives may matter, and provide a generalized rationalizability condition. Further, Rubinstein and Salant [25] studies rationalizability analysis in a general setting in which a choice problem comes in the form of a pair of a set and an arbitrary framing factor.

We consider arbitrary choice data, which may or may not be rationalizable in any sense. We are interested in how we can assign preference in a normative or prescriptive sense, rather than in the descriptive sense that individual indeed makes choice by maximizing or satisficing it.

Our model and analysis are similar to those in expected utility theory in the context of games (Gilboa-Schmeidler [13]), inductive inference (Gilboa-Schmeidler [12]), case-based 
decision making (Gilboa-Schmeidler [10]) and social aggregation of preferences (Young [33]). One technical difference, though, is that our domain is larger in the sense that choice distributions are conditioned by different situations, which makes it difficult to establish the uniqueness of the obtained individual welfare functional.

\section{Model and basic axioms}

Let $X$ be a finite set of alternatives. Let $\mathcal{B} \subset 2^{X}$ be a family of observable opportunity sets. We assume $|B| \geq 2$ for all $B \in \mathcal{B}$. For each $B \in \mathcal{B}$, let $F(B)$ be a set of possible framing factors associate with $B$, and let $F=\bigcup_{B \in \mathcal{B}} F(B)$.

Example 2 Let $B=\{x, y, z\}$. Then one may for example think of $F(B)$ being a set of linear orderings over $\{x, y, z\}$. Let $>\in F(B)$ be one such order, where $z>y>x$. Then, the pair $(B,>)$ corresponds to presenting the set $\{x, y, z\}$, in the form of an ordered triple $(z, y, x)$. This allows choice to depend on the order of appearance of alternatives.

Example 3 Let $B=\{x, y, z\}$. Then one may for example think of $F(B)=X$ being a set of reference points (in any sense). Let $a \in F(B)$ be one such reference point. Then, the pair $(B, a)$ corresponds to presenting the set $\{x, y, z\}$ together with the reference point $a$.

Let $\Theta$ be the set of observable pairs of choice opportunity and framing factors; namely, let $\Theta \subset \mathcal{B} \times F$ be defined as $\Theta=\{(B, f) \in \mathcal{B} \times F: f \in F(B)\} .{ }^{3}$ We call elements of $\Theta$ situations. Each element $\theta \in \Theta$ therefore specifies $\left(B_{\theta}, f_{\theta}\right)$ with $f_{\theta} \in F\left(B_{\theta}\right)$. Let $P=\prod_{\theta \in \Theta} \Delta\left(B_{\theta}\right)$ be the set of all possible empirical distributions of choice, where $p(x \mid \theta)$ refers to the empirical probability (relative frequency) of $x \in B_{\theta}$ being chosen in the choice situation $\theta=\left(B_{\theta}, f_{\theta}\right)$. An element in $P$ is called a situation-dependent choice distribution, or simply a choice distribution. Such empirical distributions are arbitrary, and may or may not satisfy any deterministic choice consistency conditions (Chernoff [6], Sen [28], Arrow [1]) or stochastic consistency conditions (Luce [20], Falmagne [7]). They may even violate more general conditions (Kalai, Rubinstein and Spiegler [17], Masatlioglu and Ok [23], Rubinstein and Salant [24, 25], Tyson [32]).

Remark 4 Why do we pick situation-dependent choice distributions as the primitive, rather than choice data itself? To illustrate, consider data in which the choice problem $\{x, y\}$ appears ten times and the choice problems $\{y, z\}$ and $\{x, z\}$ each appear just once. A simple kind of scoring rule, say, simply counting the number of times each alternative is chosen, likely supports $x$ and $y$ over $z$ simply because $z$ does not appear in choice problems very frequently. We avoid this type of bias due to sampling, taking the frequency of choice problems to be normalized.

\footnotetext{
${ }^{3}$ Rubinstein and Salant [25] consider a model in which a choice problem comes in the form of such pair, and investigate a rationalizability condition for choice functions defined over the extended domain.
} 
Even after normalizing the choice frequencies for each given $\theta$, there may be more $\theta$ 's with $x \in B_{\theta}$ than there are $\theta$ 's with $y \in B_{\theta}$. This issue should be resolved by appropriately weighting the pair $(x, \theta)$.

An individual welfare functional is a function that maps a situation-dependent choice distribution to a complete and transitive binary relation over $X$. Equivalently, it is given as a family of binary relations indexed by choice distributions, $\left\{\succsim_{p}\right\}_{p \in P}$, where $\succsim_{p}$ is a complete and transitive ordering over $X$ for each $p \in P$.

First, we impose the following three axioms.

Combination: For every $p, q \in P$ and $\lambda \in(0,1)$,

$$
x \succsim_{p} y \text { and } x \succsim_{q} y \text { imply } x \succsim_{\lambda p+(1-\lambda) q} y,
$$

and

$$
x \succ_{p} y \text { and } x \succsim_{q} y \text { imply } x \succ_{\lambda p+(1-\lambda) q} y .
$$

The combination axiom requires that the ranking specified by the concatenation of two choice distributions is consistent with the rankings specified by the original choice distributions. Imagine an individual who is facing the same choice situations every day. On weekdays, he behaves in one fashion, and on weekends, he behaves in another fashion. Suppose that according to the weekday data, $x$ is ranked at least as good as $y$. Also, according to the weekend data, $x$ is at least as good as $y$. Then $x$ is at least as good as $y$ overall.

Diversity: For every list of four distinct alternatives $x, y, z, w \in X$, there is $p \in P$ such that $x \succ_{p} y \succ_{p} z \succ_{p} w$.

Continuity: For every $x, y \in X$, the set $\left\{p \in P: x \succsim_{p} y\right\}$ is closed.

Continuity is also a technical requirement.

Definition 5 A series of vectors in $\mathbb{R}^{\sum_{\theta \in \Theta}\left|B_{\theta}\right|}$, denoted $\left\{u^{x}\right\}_{x \in X}$, is said to be diversified if there are no distinct four elements $x, y, z, w$ and non-negative numbers $\alpha, \beta$ and $a$ vector $r \in \mathbb{R}^{|\Theta|}$ with the property that

$$
u^{z}(\theta, b)-u^{w}(\theta, b) \geqq \alpha\left(u^{x}(\theta, b)-u^{y}(\theta, b)\right)+\beta\left(u^{y}(\theta, b)-u^{z}(\theta, b)\right)+r(\theta)
$$

and

$$
\sum_{\theta \in \Theta} r(\theta) \geqq 0
$$

or,

(ii)

$$
u^{z}(\theta, b)-u^{w}(\theta, b) \leqq \alpha\left(u^{x}(\theta, b)-u^{y}(\theta, b)\right)+\beta\left(u^{y}(\theta, b)-u^{z}(\theta, b)\right)+r(\theta)
$$

and

$$
\sum_{\theta \in \Theta} r(\theta) \leqq 0
$$


The following is our main theorem. It states that whether or not $x$ is ranked at least as good as $y$ depends linearly on the choice probabilities of every alternative in every situation. That is, for every alternative $x$, for every situation $\theta$ and every alternative $b \in B_{\theta}$ there is a real number $u^{x}(\theta, b)$ by which the probability that $b$ is chosen in situation $\theta$ is multiplied. Thus, the choice probabilities of alternatives other than $x$ and $y$ in arbitrary choice situations may be relevant for determining the ranking between $x$ and $y$. In a later section, we will explore conditions which free our individual welfare functional from such dependence. However, for now, a preference for apples over oranges may be determined by the probability that bananas are chosen.

Theorem 6 Individual welfare functional $\left\{\succsim_{p}\right\}_{p \in P}$ satisfies Combination, Diversity and Continuity if and only if there exists a diversified series of vectors in $\mathbb{R}^{\sum_{\theta \in \Theta}\left|B_{\theta}\right|}$, denoted $\left\{u^{x}\right\}_{x \in X}$, such that for every $x, y \in X$ and $p \in P$,

$$
x \succsim_{p} y \Longleftrightarrow \sum_{\theta \in \Theta} \sum_{b \in B_{\theta}} u^{x}(\theta, b) p(b \mid \theta) \geqq \sum_{\theta \in \Theta} \sum_{b \in B_{\theta}} u^{y}(\theta, b) p(b \mid \theta) .
$$

Moreover, when another series of vectors $\left\{v^{x}\right\}_{x \in X}$ satisfies the above condition, there is a positive scaler $\lambda$ and a series of vectors $\left\{\beta^{x}\right\}_{x \in X}$ in $\mathbb{R}^{\sum_{\theta \in \Theta}\left|B_{\theta}\right|}$

$$
v^{x}(\theta, b)=\lambda u^{x}(\theta, b)+\beta^{x}(\theta, b),
$$

where for all $x, y \in X$ :

(i) $\beta^{x}(\theta, b)-\beta^{y}(\theta, b)=\beta^{x}\left(\theta, b^{\prime}\right)-\beta^{y}\left(\theta, b^{\prime}\right)$ for all $\theta \in \Theta, b, b^{\prime} \in B_{\theta}$;

(ii) $\sum_{\theta \in \Theta} \sum_{b \in B_{\theta}} \beta^{x}(\theta, b)=\sum_{\theta \in \Theta} \sum_{b \in B_{\theta}} \beta^{y}(\theta, b)$.

\section{Binary Independence}

\subsection{Binary Independence}

Thus far, we have been concerned only with the combination axiom; and our resulting representation theorem is very general. This is because we have required no axiom to rule out dependence of the ranking of a pair of alternatives from the choice probabilities of other alternatives. In this section, we propose just such a notion. The axiom of binary independence states formally that a ranking depends only on the probabilities of the alternatives under consideration. Binary monotonicity states that this ranking should somehow depend positively on these probabilities; that is, if $x$ is ranked above $y$ for one distribution, and the probability of $x$ only increases to another distribution (where the probability of $y$ may decrease), then we should not claim that $y$ is ranked above $x$ for the new distribution.

Binary Independence/Monotonicity: For every $x, y \in X$ and $p, q \in P$ :

(i) if $p(x \mid \theta)=q(x \mid \theta)$ for all $\theta \in \Theta$ with $x \in B_{\theta}$ and $p(y \mid \theta)=q(y \mid \theta)$ for all $\theta \in \Theta$ with $y \in B_{\theta}$, then $x \succsim_{p} y$ if and only if $x \succsim_{q} y$;

(ii) if $x \succsim_{p} y$ and $p(x \mid \theta) \leqq q(x \mid \theta)$ for all $\theta \in \Theta$ with $x \in B_{\theta}$ and $p(y \mid \theta) \geqq q(y \mid \theta)$ for all $\theta \in \Theta$ with $y \in B_{\theta}$, and at least one of these inequalities are strict, then $x \succ_{q} y$. 


\subsection{Result on the domain of multinomial choices}

In this subsection, we assume that $\Theta_{x} \neq \emptyset$ for all $x \in X$, and $\left|B_{\theta}\right| \geqq 3$ for all $\theta \in \Theta$.

The next property states that any alternatives which are never chosen should be ranked indifferent.

Dummy: For every $x, y \in X$, if $p \in P$ is such that $p(x \mid \theta)=0$ for all $\theta \in \Theta$ with $x \in B_{\theta}$ and $p(y \mid \theta)=0$ for all $\theta \in \Theta$ with $y \in B_{\theta}$, then $x \sim_{p} y$.

For each $x \in X$, let $\Theta_{x}=\left\{\theta \in \Theta: B_{\theta} \ni x\right\}$.

Definition $7 A$ series of vectors $\left\{u^{x}\right\}_{x \in X}$, where $u^{x}$ is an element in $\mathbb{R}_{++}^{\left|\Theta_{x}\right|}$ for each $x$, is said to be diversified if the series of vectors $\widetilde{u}^{x}$ in $\mathbb{R}^{\sum_{\theta \in \Theta}\left|B_{\theta}\right|}$, which is given by $\widetilde{u}^{x}(\theta, x)=u^{x}(\theta)$ for each $\theta \in \Theta_{x}$ and zero elsewhere, is diversified.

The next theorem is similar to Theorem 6, however, the utility representation of the induced ranking states that the utility of an alternative depends only on the choice probabilities of that alternative.

Theorem 8 Assume that $\Theta_{x} \neq \emptyset$ for all $x \in X$, and $\left|B_{\theta}\right| \geqq 3$ for all $\theta \in \Theta$. Then, an individual welfare functional $\left\{\succsim_{p}\right\}_{p \in P}$ satisfies Combination, Diversity, Continuity, Binary Independence/Monotonicity and Dummy, if and only if there exists a diversified series of strictly positive vectors $\left\{u^{x}\right\}_{x \in X}$, where $u^{x}$ is an element in $\mathbb{R}_{++}^{\left|\Theta_{x}\right|}$ for each $x$, such that for all $x, y \in X$,

$$
x \succsim_{p} y \Longleftrightarrow \sum_{\theta \in \Theta_{x}} u^{x}(\theta) p(x \mid \theta) \geqq \sum_{\theta \in \Theta_{y}} u^{y}(\theta) p(y \mid \theta)
$$

for every $p \in P$.

Moreover, $\left\{u^{x}\right\}_{x \in X}$ is unique up to common positive scaler multiplications.

The following example shows that the diversity axiom is independent of the other axioms used in the characterization.

Example 9 Let $X=\{x, y, z, w\}$ and $\Theta=\left\{\theta_{1}, \theta_{2}\right\}$, where $B_{\theta_{1}}=\{x, y, z\}$ and $B_{\theta_{2}}=$ $\{z, y, w\}$. Consider the scoring rule given by

$$
U(x)=p(x \mid\{x, y, z\})+p(x \mid\{x, y, w\}), U(y)=p(y \mid\{x, y, z\})+p(y \mid\{x, y, w\})
$$

and

$$
U(z)=p(z \mid\{x, y, z\}), U(w)=p(w \mid\{x, y, w\}) .
$$

Then, $x \succsim_{p} w$ and $y \succsim_{p} w$ always imply $w \succsim_{p} z$, which cannot satisfy the diversity axiom. 


\subsection{Result on the domain including binary choices}

For technical reasons, when the choice situations can involve binary choices, a few more axioms are needed to characterize individual welfare functionals as in Theorem 8. To see why this is the case, imagine a domain in which all choice situations involve only binary choices. Then binary independence/monotonicity is clearly vacuous, and extra structure is needed. The axioms in this section deliver this extra structure.

Given $p \in P, \theta \in \Theta$ and $q_{\theta} \equiv q(\cdot \mid \theta) \in \Delta\left(B_{\theta}\right)$, let $\left(q_{\theta}, p_{-\theta}\right)$ be the situation dependent choice distribution that coincides with $q_{\theta}$ at $\theta$ and with $p$ elsewhere.

Proportionality: For every $x, y, z \in X$ and $p \in X$ such that $y \sim_{p} z \succ_{p} x$, any $\theta \in \Theta$ with $B_{\theta}=\{x, y\}$, and $q_{\theta} \in \Delta\left(B_{\theta}\right)$,

if

$$
x \sim(1-\lambda) p+\lambda\left(q_{\theta}, p_{-\theta}\right) z
$$

then

$$
x \sim\left(1-\frac{\lambda}{2}\right) p+\frac{\lambda}{2}\left(q_{\theta}, p_{-\theta}\right) y
$$

Strong Dummy: For every $x, y \in X$, if $p \in P$ is such that $p(x \mid \theta)=0$ for all $\theta \in \Theta$ with $x \in B_{\theta}$ and $B_{\theta} \neq\{x, y\}, p(y \mid \theta)=0$ for all $\theta \in \Theta$ with $y \in B_{\theta}$ and $B_{\theta} \neq\{x, y\}$, and $p(x \mid \theta)=p(y \mid \theta)$ for all $\theta \in \Theta$ with $B_{\theta}=\{x, y\}$, then $x \sim_{p} y$.

Also, let $\Theta_{\{x, y\}}=\left\{\theta \in \Theta: B_{\theta}=\{x, y\}\right\}$.

Theorem 10 Assume that $\Theta_{x} \neq \emptyset$ for all $x \in X$. Then, an individual welfare functional $\left\{\succsim_{p}\right\}_{p \in P}$ satisfies Combination, Diversity, Continuity, Binary Independence/Monotonicity, Proportionality and Strong Dummy if and only if there exists a diversified series of strictly positive vectors $\left\{u^{x}\right\}_{x \in X}$, where $u^{x}$ is an element in $\mathbb{R}_{++}^{\left|\Theta_{x}\right|}$ for each $x$, such that for all $x, y \in X$,

$$
x \succsim_{p} y \Longleftrightarrow \sum_{\theta \in \Theta_{x}} u^{x}(\theta) p(x \mid \theta) \geqq \sum_{\theta \in \Theta_{y}} u^{y}(\theta) p(y \mid \theta)
$$

for every $p \in P$, and $\sum_{\theta \in \Theta_{\{x, y\}}} u^{x}(\theta)=\sum_{\theta \in \Theta_{\{x, y\}}} u^{y}(\theta)$ holds.

Moreover, $\left\{u^{x}\right\}_{x \in X}$ is unique up to common positive scaler multiplications.

The following example shows that the diversity axiom may not follow from the others.

Example 11 Let $X=\{x, y, z, w\}$ and $\Theta=\left\{\theta_{1}, \theta_{2}\right\}$, where $B_{\theta_{1}}=\{x, y\}$ and $B_{\theta_{2}}=$ $\{z, w\}$. Consider the scoring rule given by

$$
U(x)=p(x \mid\{x, y\}), U(y)=p(y \mid\{x, y\}), U(z)=p(z \mid\{z, w\}), U(w)=p(w \mid\{z, w\}) .
$$

Then, we have $x \succsim_{p} z$ if and only if $w \succsim_{p} y$, which cannot satisfy the diversity axiom. 


\section{Narrowing down the weights}

We are mostly concerned with a formal consistency requirement or informational efficiency requirement about how to map choice data into a preference, and away from substantive views about which weights should be used. Of course, the Diversity and Dummy axioms rule out certain lists of potential weights.

Here we propose several ways to narrow down the weights, in order to respect choice and eliminate unnecessary or unjustified paternalism. The idea is that one should avoid putting a larger prior weight on a particular alternative without any sufficient reason. Of course, what is meant by 'sufficient' reason depends on what kind of choice frames are in present. Hence we do it case by case.

\subsection{Weights on opportunities}

Our representation theorems allow us to weight situations by how many elements are available. That is, the economist may have a certain prior weight about how much being chosen from a larger set is more (or less) important.

For simplicity, here we assume that for all $B \in \mathcal{B}, F(B)$ is a singleton; so that $\Theta$ may be formally identified with a class of elements of $\mathcal{B}$; without loss of generality, assume $\Theta \subset \mathcal{B}$. For all permutations $\pi: X \rightarrow X$ and for all $B \in \Theta$, we require that $\pi(B) \in \Theta$. For $p \in P$, the probability of $x$ being chosen from $B$ is written as $p(x \mid B)$.

Given $p \in P$ and $\pi$, a permutation over $X$, define $p^{\pi}$ by

$$
p^{\pi}(x \mid B)=p\left(\pi^{-1}(x) \mid \pi^{-1}(B)\right) .
$$

Neutrality: For all $p \in P, x, y \in X$ and any permutation $\pi$ over $X$,

$$
x \succsim_{p} y \Longleftrightarrow \pi(x) \succsim_{p^{\pi}} \pi(y)
$$

Corollary 12 An individual welfare functional as characterized in Theorem 8 or 10 further satisfies Neutrality if and only if there exists a function $u:\{|B|: B \in \mathcal{B}\} \rightarrow \mathbb{R}_{++}$ such that

$$
u^{x}(B)=u\left(\left|B_{\theta}\right|\right)
$$

for all $x \in X$ and $B \in \mathcal{B}$ with $x \in B$.

One may here give a solution to the problem in the leading example. Being chosen from larger sets is more important whenever $u$ is increasing. Being chosen in direct comparisons is of highest importance when $u(2)>u(k)$ for all $k \neq 2$.

This individual welfare functional respects choice. For simplicity, assume $\Theta=\{B \in$ $\left.2^{X}:|B| \geqq 2\right\}$. Suppose now that there exists a strict preference $\succ$ such that $x_{1} \succ x_{2} \succ$ 
$\cdots \succ x_{n}$, and that choice is made according to this ranking in a deterministic manner. Then, the score given to alternative $x_{k}$, which is the $k$-th best element, is

$$
U\left(x_{k}\right)=\sum_{l=2}^{n-k+1} C(n-k, l-1) u(l) .
$$

This is obviously decreasing in $k$, and the assigned ranking agrees with $\succ$, whatever $u$ is. Here $u$ has nothing to say.

\subsection{Weights on framing factors}

Let $F(B)$ be the set of linear orderings over $B$, which describe possible orders of appearance. Given $B \in \mathcal{B}$, an order $>\in F(B)$, and $x \in B$, let $r(x, B,>)$ be the rank of $x$ in $B$ with regard to the order $>$. For example, $r(x, B,>)=1$ if $x$ is the first element to be presented from $B$ according to $>$, and $r(x, B,>)=|B|$ if it is the last one.

In this case, one can impose additional structure to the previous example, so as to incorporate weights on framing, by writing $u^{x}(\theta)$ as a function of $\left|B_{\theta}\right|$ and $r\left(x, B_{\theta},>_{\theta}\right)$, say $u^{x}(\theta)=u^{\prime}\left(\left|B_{\theta}\right|, r\left(x, B_{\theta},>_{\theta}\right)\right.$. We believe it is sensible for the function $u^{\prime}$ to be nondecreasing in its second argument. This might be because it an alternative which is listed in the back is less likely to be chosen, so that being chosen nevertheless is valued more. ${ }^{4}$

\subsection{Adjusting to the number of appearance}

When some alternative appears in more situations than others, that is, when $\left|\Theta_{x}\right|$ is not equal across $x$ 's, one may worry about the possibility that $x$ is valued highly simply because of this. The class of individual welfare functionals we obtain allows one to adjust weights according to how many choice problems in which an alternative appears, by letting for example

$$
u^{x}(\theta)=\frac{u^{\prime}\left(x, B_{\theta}, f_{\theta}\right)}{\left|\Theta_{x}\right|} \text { for all } x \in X, \theta \in \Theta_{x}
$$

\section{Concluding comments}

In the paper we have discussed how to prescribe a preference ranking for a given individual based on observed choices when these choices may be inconsistent. We consider a function that maps choice data (a situation dependent empirical distribution) into a complete and transitive ordering over alternatives.

\footnotetext{
${ }^{4}$ Though, it also allows that $u$ has a sudden drop or spike, when for example being the last one has a focal-point effect.
} 
The main axiom we impose is Combination, which basically says if both of two choice data respectively support $x$ over $y$, then the choice data obtained by joining the two also supports $x$ over $y$.

Together with other mild axioms, we obtain a scoring type rule, where we have to have a prior view about which choice situation is more (or less) important in forming the preference that should be assigned.

The additional axioms, Binary Independence above all, deliver a more structured class of scoring rules, where the score for a given alternative depends only on its empirical choice probability.

However, the role of such weights is only in balancing inconsistent choices, and has nothing to say when choices are indeed consistent.

\subsection{Directions for future research}

Our work serves as a first approach to the problem of evaluating welfare when choice data is inconsistent. There are several obvious paths to follow in this direction.

Firstly, we have throughout assumed that our individual welfare functional satisfies Diversity. This condition requires that the output of an individual welfare functional is non-imposed on the individual who makes choices. It is similar to the citizen's sovereignty requirement of social choice. In the interest of libertarianism, such a requirement is natural. However, in order to allow our individual welfare functional to be more paternalistic, we may wish to force certain preferences on individuals. For example, many individuals may choose to start consuming an addictive substance, and once they start consuming they never stop. Our notion of individual welfare functional would then say that the consumption of this substance makes the individual better off. However, such an individual may not feel better off in the long run. We might therefore imagine a program which leads this individual to be addicted to the substance to be a bad one, even though it is one which he himself would choose. Our axioms at this stage do not allow such recommendations.

Secondly, there is a question of time-dependence. Individuals may make repeated choices, say between $x$ and $y$. In an environment where they have never before consumed these types of alternatives, they must experiment. Suppose we observe an individual who has consumed $x$ in the first five periods, but then consumed $y$ in the last five. It seems plausible to suspect that this individual has learned that they prefer $y$ to $x$. However, the primitive of our model (a probability distribution over choices) does not allow us to make such conclusions.

Lastly, there is the question as to what rationality actually means. Our work has taken as given that rationality is rationalizability by a complete and transitive preference. However, weaker (and stronger) notions of rationality certainly exist. In more structured 
environments, for example, in the theory of choice over lotteries, satisfaction of the independence axiom is often understood as a basic tenet of rationality. This is an issue of the specification of the range of our individual welfare functional (related is the issue of domain specification; however it seems natural to allow our individual welfare functional to take as input any stochastic choice function).

\section{Proofs}

\subsection{Proof of Theorem 6}

Given $p \in P$ and a vector $u \in \mathbb{R}^{\sum_{\theta \in \Theta}\left|B_{\theta}\right|}$, let

$$
u \cdot p=\sum_{\theta \in \Theta} \sum_{b \in B_{\theta}} u(\theta, b) p(b \mid \theta)
$$

Lemma 13 There exists a series of vectors in $\mathbb{R}^{\sum_{\theta \in \Theta}\left|B_{\theta}\right|}$, denoted $\left\{u^{x y}\right\}_{x, y \in X, x \neq y}$, such that for every $x, y \in X$,

(i) $\left\{p \in P: u^{x y} \cdot p \geqq 0\right\}=\left\{p \in P: x \succsim_{p} y\right\}$;

(ii) $\left\{p \in P: u^{x y} \cdot p>0\right\}=\left\{p \in P: x \succ_{p} y\right\}$;

(iii) $\left\{p \in P: u^{x y} \cdot p \leqq 0\right\}=\left\{p \in P: y \succsim_{p} x\right\}$;

(iv) $\left\{p \in P: u^{x y} \cdot p<0\right\}=\left\{p \in P: y \succ_{p} x\right\}$;

(v) neither $u^{x y} \geqq 0$ or $u^{x y} \leqq 0$;

(vi) $u^{y x}=-u^{x y}$.

Moreover, if another vector series of vectors $\left\{v^{x y}\right\}_{x, y \in X, x \neq y}$ delivers the above properties, then there is a series of positive numbers $\left\{\lambda^{x y}\right\}_{x, y \in X, x \neq y}$ and a series of vectors in $\mathbb{R}^{|\Theta|}$, denoted $\left\{r^{x y}\right\}_{x, y \in X, x \neq y}$, such that for every $x, y \in X$,

$$
v^{x y}(\theta, b)=\lambda^{x y} u^{x y}(\theta, b)+r^{x y}(\theta)
$$

for all $\theta \in \Theta$ and $b \in B_{\theta}$;

(2) $\sum_{\theta \in \Theta} r^{x y}(\theta)=0$;

(3) $\lambda^{x y}=\lambda^{y x}$

(4) $r^{y x}=-r^{x y}$.

Proof: Existence: Fix $x, y \in X$, and let $C^{x y}=\left\{p \in P: x \succsim_{p} y\right\}$ and $C^{y x}$ similarly. By the diversity axiom and continuity axiom, both have nonempty interiors relative to $P$, and relint $C^{x y}=\left\{p \in P: x \succ_{p} y\right\}$ holds. By completeness, $C^{x y} \cup C^{y x}=P$. Also, we have $C^{x y} \cup$ relint $C^{y x}=\emptyset$ and similarly for the reverse combination.

Claim 14 If $d, e \in \Phi^{x y}$, then $d+e \in \Phi^{x y}$.

Proof: Without loss of generality, let $d, e \in C^{x y}-C^{y x}$, and let $d=p_{1}-p_{2}$ and $e=q_{1}-q_{2}$, where $p_{1}, q_{1} \in C^{x y}$ and $p_{2}, q_{2} \in C^{y x}$. By the combination axiom, $\frac{p_{1}+q_{1}}{2} \in C^{x y}$ and $\frac{p_{2}+q_{2}}{2} \in C^{y x}$. Therefore $\frac{d+e}{2}=\frac{p_{1}+q_{1}}{2}-\frac{p_{2}+q_{2}}{2} \in C^{x y}-C^{y x}$. Therefore $d+e \in \Phi^{x y}$. 
Let $\Phi^{x y}=\left\{\lambda(p-q): \lambda \geqq 0, p \in C^{x y}, q \in C^{y x}\right\}$, and define a binary relation $\succsim^{x y}$ over $P$ by

$$
p \succsim^{x y} q \text { if } p-q \in \Phi^{x y}
$$

Since $C^{x y}$ and $C^{y x}$ are compactly generated cones by continuity, $\Phi^{x y}$ is closed, hence $\succsim^{x y}$ is a closed binary relation.

Claim $15 \succsim^{x y}$ is complete.

Proof: Case 1: The claim is obvious when $p \in C^{x y}$ and $q \in C^{y x}$.

Case 2: Suppose $p, q \in C^{x y}$. Pick any $r \in$ relint $C^{y x}$. Then, there exists $\lambda \in[0,1)$ such that both $(1-\lambda) p+\lambda r$ and $(1-\lambda) q+\lambda r$ are in $C^{x y}$ and at least one of them is in $\partial C^{x y}=C^{x y} \cap C^{y x}$. Without loss, suppose $(1-\lambda) q+\lambda r \in \partial C^{x y}$, then we have

$$
[(1-\lambda) p+\lambda r]-[(1-\lambda) q+\lambda r]=(1-\lambda)(p-q) \in \Phi^{x y}
$$

which implies $p-q \in \Phi^{x y}$.

Case 3: Similarly for the case that $p, q \in C^{y x}$.

Claim $16 \succsim^{x y}$ is transitive.

Proof: Suppose $p \succsim^{x y} q$ and $q \succsim^{x y} r$. Since $p-q \in \Phi^{x y}$ and $q-r \in \Phi^{x y}$, from the previous claim we have $p-r=(p-q)+(q-r) \in \Phi^{x y}$.

Claim 17 For every $p, q, r \in \mathcal{P}$ and $\lambda \in(0,1), p \succsim^{x y} q$ if and only if $\lambda p+(1-\lambda) r \succsim^{x y}$ $\lambda q+(1-\lambda) r$.

Proof: It follows from $(\lambda p+(1-\lambda) r)-(\lambda q+(1-\lambda) r)=\lambda(p-q)$.

Since $\succsim^{x y}$ satisfies the state-dependent version of Anscombe-Aumann subjective expected utility theorem (see Kreps [19], Karni-Schmeidler-Vind [18]), there is a vector $\widetilde{u}^{x y} \in \mathbb{R}^{\sum_{\theta \in \Theta}\left|B_{\theta}\right|}$ such that

$$
p \succsim^{x y} q \Longleftrightarrow \widetilde{u}^{x y} \cdot p \geqq \widetilde{u}^{x y} \cdot q
$$

for every $p, q \in P$.

Moreover, the vector $\widetilde{u}^{x y}$ is unique in the sense that if there is another vector $\widetilde{v}^{x y}$ that delivers the above condition then there exist a positive number $\lambda^{x y}$ and a vector $\widetilde{r}^{x y} \in \mathbb{R}^{|\Theta|}$ such that $\widetilde{v}^{x y}(\theta, b)=\lambda^{x y} \widetilde{u}^{x y}(\theta, b)+\widetilde{r}^{x y}(\theta)$ for every $\theta \in \Theta$ and $b \in B_{\theta}$.

Fix any $p^{*} \in C^{x y} \cap C^{y x}$, and let $\alpha^{x y}=\widetilde{u}^{x y} \cdot p^{*}$. Define a vector $u^{x y} \in \mathbb{R}^{\sum_{\theta \in \Theta}\left|B_{\theta}\right|}$ by $u^{x y}(\theta, b)=\widetilde{u}^{x y}(\theta, b)-\frac{\alpha^{x y}}{|\Theta|}$ for every $\theta$ and $b \in B_{\theta}$. Then, it satisfies

$$
u^{x y} \cdot p \geqq 0 \Longleftrightarrow x \succsim_{p} y
$$


for all $p \in P$. This delivers the properties (i)-(iv).

To show property (v), suppose $u^{x y} \geqq 0$. Then, since $u^{x y} \cdot p \geqq 0$ for all $p \in P$, we have $x \succsim_{p} y$ for all $p \in P$, which is a contradiction to diversity. The similar contradiction is obtained for the case $u^{x y} \leqq 0$.

Uniqueness: Suppose $v^{x y}$ satisfies

$$
v^{x y} \cdot p \geqq 0 \Longleftrightarrow x \succsim_{p} y
$$

for all $p \in P$. Then, $v^{x y}$ also forms a representation for $\succsim^{x y}$, where $v^{x y} \cdot p^{*}=0$ holds for $p^{*} \in C^{x y} \cap C^{y x}$. By the uniqueness of representation of $\succsim^{x y}$ above, there exist a positive number $\lambda^{x y}$ and a vector $\widetilde{r}^{x y} \in \mathbb{R}^{|\Theta|}$ such that

$$
\begin{aligned}
v^{x y}(\theta, b) & =\lambda^{x y} \widetilde{u}^{x y}(\theta, b)+\widetilde{r}^{x y}(\theta) \\
& =\lambda^{x y}\left(u^{x y}(\theta, b)-\frac{\alpha^{x y}}{|\Theta|}\right)+\widetilde{r}^{x y}(\theta) \\
& =\lambda^{x y} u^{x y}(\theta, b)+r^{x y}(\theta)
\end{aligned}
$$

for every $\theta \in \Theta$ and $b \in B_{\theta}$, where $r^{x y}$ is defined by $r^{x y}(\theta)=\widetilde{r}^{x y}(\theta)-\frac{\alpha^{x y}}{|\Theta|}$ for each $\theta$. Since $v^{x y} \cdot p^{*}=u^{x y} \cdot p^{*}=0$, we have $\sum_{\theta \in \Theta} r^{x y}(\theta)=0$.

Lemma 18 For every distinct $x, y, z \in X$ and $\varepsilon, \eta \in \mathbb{R}$, if $\varepsilon u^{x y}+\eta u^{y z} \leqq 0$, then $\varepsilon=\eta=0$.

Proof: Since neither $u^{x y} \geqq 0$ or $u^{x y} \leqq 0$ holds, if either of $\varepsilon$ and $\eta$ is zero, so is the other. Suppose both are non-zero. Without loss of generality, suppose that $\varepsilon, \eta>0$. Then, we have $\varepsilon u^{y x} \leqq-\eta u^{y z}=\eta u^{z y}$. Therefore, $z \succsim_{p} y$ implies $y \succsim_{p} x$, contradicting Diversity. Similar contradictions are obtained for the other cases.

Lemma 19 Let $\left\{u^{x y}\right\}_{x, y \in X, x \neq y}$ be the series of vectors obtained in the previous lemma. Then, for every three distinct $x, y, z$, there are unique number $\alpha, \beta>0$ and $r \in \mathbb{R}^{|\Theta|}$ such that

$$
u^{x z}(\theta, b)=\alpha u^{x y}(\theta, b)+\beta u^{y z}(\theta, b)+r(\theta)
$$

for all $\theta \in \Theta$ and $b \in B_{\theta}$, and

$$
\sum_{\theta \in \Theta} r(\theta)=0
$$

Proof: As for all $p \in P, \succsim_{p}$ is transitive, there does not exist $p \in P$ such that

$$
u^{x y} \cdot p \geqq 0, u^{y z} \cdot p \geqq 0 \text { and }-u^{x z} \cdot p>0 .
$$

For each $\theta \in \Theta$, let $\mathbf{1}_{\theta} \in \mathbb{R}^{\sum_{\theta \in \Theta}\left|B_{\theta}\right|}$ be that vector whose coordinate is 1 at all $(\theta, \cdot)$ and 0 elsewhere. Also, for each $\theta \in \Theta$ and $b \in B_{\theta}$, let $\mathbf{1}_{\theta, b} \in \mathbb{R}^{\sum_{\theta \in \Theta}\left|B_{\theta}\right|}$ be that vector whose coordinate is 1 only at $(\theta, b)$ and 0 elsewhere. 
By a nonhomogeneous version of the Farkas' lemma (see Proposition 4.2.3 of [8]), there exist non-negative numbers $\alpha, \beta,\left\{\lambda_{\theta, b}\right\}_{\theta \in \Theta, b \in B_{\theta}},\left\{\mu_{\theta}\right\}_{\theta \in \Theta}$ and $\left\{\nu_{\theta}\right\}_{\theta \in \Theta}$ such that

$$
u^{x z}=\alpha u^{x y}+\beta u^{y z}+\sum_{\theta \in \Theta} \sum_{b \in B_{\theta}} \lambda_{\theta, b} \mathbf{1}_{\theta, b}+\sum_{\theta \in \Theta} \mu_{\theta} \mathbf{1}_{\theta}-\sum_{\theta \in \Theta} \nu_{\theta} \mathbf{1}_{\theta}
$$

and

$$
\sum_{\theta \in \Theta} \mu_{\theta}-\sum_{\theta \in \Theta} \nu_{\theta} \geqq 0
$$

Hence,

$$
u^{x z} \geqq \alpha u^{x y}+\beta u^{y z}+\sum_{\theta \in \Theta} \mu_{\theta} \mathbf{1}_{\theta}-\sum_{\theta \in \Theta} \nu_{\theta} \mathbf{1}_{\theta} .
$$

Note that for all $p \in P,\left(\sum_{\theta \in \Theta} \mu_{\theta} \mathbf{1}_{\theta}-\sum_{\theta \in \Theta} \nu_{\theta} \mathbf{1}_{\theta}\right) \cdot p \geqq 0$.

Consequently, if $\alpha=0$, then for all $p \in P, y \succsim_{p} z$ implies $x \succsim_{p} z$, contradicting Diversity. Hence $\alpha>0$. Similarly, $\beta>0$.

A symmetric argument applied to $-u^{x y},-u^{y z}$ and $u^{x z}$ delivers $\delta, \gamma>0$ and nonnegative numbers $\left\{\rho_{\theta}\right\}_{\theta \in \Theta}$ and $\left\{\tau_{\theta}\right\}_{\theta \in \Theta}$ such that

$$
u^{x z} \leqq \delta u^{x y}+\gamma u^{y z}+\sum_{\theta \in \Theta} \rho_{\theta} \mathbf{1}_{\theta}-\sum_{\theta \in \Theta} \tau_{\theta} \mathbf{1}_{\theta}
$$

, where

$$
\sum_{\theta \in \Theta} \rho_{\theta}-\sum_{\theta \in \Theta} \tau_{\theta} \leqq 0
$$

By taking the sum of these inequalities, we have

$$
\left(\sum_{\theta \in \Theta} \rho_{\theta} \mathbf{1}_{\theta}-\sum_{\theta \in \Theta} \tau_{\theta} \mathbf{1}_{\theta}\right)-\left(\sum_{\theta \in \Theta} \mu_{\theta} \mathbf{1}_{\theta}-\sum_{\theta \in \Theta} \nu_{\theta} \mathbf{1}_{\theta}\right) \geqq(\alpha-\delta) u^{x y}+(\beta-\gamma) u^{y z}
$$

Therefore, for all $p \in P$,

$$
0 \geqq(\alpha-\delta) u^{x y} \cdot p+(\beta-\gamma) u^{y z} \cdot p .
$$

By the same logic as in the previous lemma, appealing to Diversity, we conclude $\alpha=\delta$ and $\beta=\gamma$. This further implies that

$$
\sum_{\theta \in \Theta} \mu_{\theta}-\sum_{\theta \in \Theta} \nu_{\theta}=\sum_{\theta \in \Theta} \rho_{\theta}-\sum_{\theta \in \Theta} \tau_{\theta}=0
$$

Then define $r(\theta)=\mu_{\theta}-\nu_{\theta}$, for example. 
Define $\bar{u}^{x y}$ and $\bar{u}^{y z}$ by

$$
\bar{u}^{x y}(\theta, b)=\alpha u^{x y}(\theta, b)+\frac{\alpha}{\alpha+\beta} r(\theta), \bar{u}^{y z}(\theta, b)=\alpha u^{y z}(\theta, b)+\frac{\alpha}{\alpha+\beta} r(\theta),
$$

and rename them by $u^{x y}$ and $u^{y z}$, respectively. Repeat such scalar multiplications and translations (one can finish it in finite steps since $X$ is finite). As is true in GilboaSchmeidler [11], we need the full diversity condition for four elements here. Consequently we obtain

Lemma 20 There exist a series of vectors $\left\{u^{x y}\right\}_{x, y \in X, x \neq y}$, as in Lemma 13, such that for every three distinct $x, y, z$ the condition $u^{x y}+u^{y z}=u^{x z}$ holds.

Moreover, when another series of vectors $\left\{v^{x y}\right\}_{x, y \in X, x \neq y}$ satisfies the above condition, there is a positive number $\lambda$ and a series of vectors in $\mathbb{R}^{|\Theta|}$, denoted $\left\{r^{x y}\right\}_{x, y \in X, x \neq y}$, such that for every $x, y, z \in X$,

$$
v^{x y}(\theta, b)=\lambda u^{x y}(\theta, b)+r^{x y}(\theta)
$$

for all $\theta \in \Theta$ and $b \in B_{\theta}$;

(2) $\sum_{\theta \in \Theta} r^{x y}(\theta)=0$;

(3) $r^{y x}=-r^{x y}$;

(4) $r^{x y}+r^{y z}=r^{x z}$.

Proofs about diversity

First we show that the diversity condition implies the desired property.

The diversity condition implies that for all distinct $x, y, z, w \in X$, there is $p \in P$ such that

$$
u^{x y} \cdot p \geqq 0, u^{y z} \cdot p \geqq 0, u^{z w} \cdot p \geqq 0
$$

By Proposition 4.2.3 of [8], there do not exist non-negative numbers $\alpha, \beta$, $\left\{\lambda_{\theta, b}\right\}_{\theta \in \Theta, b \in B_{\theta}},\left\{\mu_{\theta}\right\}_{\theta \in \Theta}$ and $\left\{\nu_{\theta}\right\}_{\theta \in \Theta}$ such that

$$
u^{z w}=\alpha u^{x y}+\beta u^{y z}+\sum_{\theta \in \Theta} \sum_{b \in B_{\theta}} \lambda_{\theta, b} \mathbf{1}_{\theta, b}+\sum_{\theta \in \Theta} \mu_{\theta} \mathbf{1}_{\theta}-\sum_{\theta \in \Theta} \nu_{\theta} \mathbf{1}_{\theta}
$$

and

$$
\sum_{\theta \in \Theta} \mu_{\theta}-\sum_{\theta \in \Theta} \nu_{\theta} \geqq 0
$$

This implies that there cannot exist non-negative numbers $\alpha, \beta$ and a vector $r \in \mathbf{R}^{|\Theta|}$ such that

$$
u^{z w}(\theta, b) \geqq \alpha u^{x y}(\theta, b)+\beta u^{y z}(\theta, b)+r(\theta)
$$

and

$$
\sum_{\theta \in \Theta} r(\theta) \geqq 0
$$


Likewise, there cannot exist non-negative numbers $\alpha, \beta$ and a vector $r \in \mathbf{R}^{|\Theta|}$ such that

$$
u^{z w}(\theta, b) \leqq \alpha u^{x y}(\theta, b)+\beta u^{y z}(\theta, b)+r(\theta)
$$

and

$$
\sum_{\theta \in \Theta} r(\theta) \leqq 0
$$

Now we show that the diversity condition follows from the property of $\left\{u^{x y}\right\}_{x, y \in X}, x \neq$ $y$. Suppose the diversity condition is not met, then there does not exist $p \in P$ such that

$$
u^{x y} \cdot p>0, u^{y z} \cdot p>0, u^{z w} \cdot p>0 .
$$

In other words, for all $p \in P$

$$
u^{x y} \cdot p>0, u^{y z} \cdot p>0 \Longrightarrow-u^{z w} \cdot p \geqq 0 .
$$

By a continuity argument, we have that for all $p \in P$,

$$
u^{x y} \cdot p \geqq 0, u^{y z} \cdot p \geqq 0 \Longrightarrow-u^{z w} \cdot p \geqq 0 \text {. }
$$

By Proposition 4.2.3 of [8], there exist non-negative numbers $\alpha, \beta,\left\{\lambda_{\theta, b}\right\}_{\theta \in \Theta, b \in B_{\theta}}$, $\left\{\mu_{\theta}\right\}_{\theta \in \Theta}$ and $\left\{\nu_{\theta}\right\}_{\theta \in \Theta}$ such that

$$
-u^{z w}=\alpha u^{x y}+\beta u^{y z}+\sum_{\theta \in \Theta} \sum_{b \in B_{\theta}} \lambda_{\theta, b} \mathbf{1}_{\theta, b}+\sum_{\theta \in \Theta} \mu_{\theta} \mathbf{1}_{\theta}-\sum_{\theta \in \Theta} \nu_{\theta} \mathbf{1}_{\theta}
$$

and

$$
\sum_{\theta \in \Theta} \mu_{\theta}-\sum_{\theta \in \Theta} \nu_{\theta} \geqq 0
$$

However, this contradicts Diversity.

Establishing the representation

Now, fix $e \in X$, and define $u^{e} \equiv \mathbf{0} \in \mathbb{R}^{\sum_{\theta \in \Theta}\left|B_{\theta}\right|}$, and for any other $x \in X$, define $u^{x}=u^{x e}$.

Given $p \in P$ and $x, y \in X$, we have

$$
\begin{aligned}
x \succsim_{p} y & \Longleftrightarrow u^{x y} \cdot p \geqq 0 \Longleftrightarrow\left(u^{x e}+u^{e y}\right) \cdot p \geqq 0 \Longleftrightarrow\left(u^{x e}-u^{y e}\right) \cdot p \geqq 0 \\
& \Longleftrightarrow u^{x} \cdot p \geqq u^{y} \cdot p .
\end{aligned}
$$

Uniqueness: Take the representation $\left\{u^{x}\right\}_{x \in X}$ constructed above, where $u^{e}=\mathbf{0}$. Suppose another series of vectors $\left\{v^{x}\right\}_{x \in X}$ satisfies the above condition. Then, the series of vectors $\left\{v^{x}-v^{y}\right\}_{x, y \in X, x \neq y}$ satisfies the condition in the previous lemma. Hence there is a scaler $\lambda$ 
and a a series of vectors in $\mathbb{R}^{|\Theta|}$, denoted $\left\{r^{x y}\right\}_{x, y \in X, x \neq y}$, such that for every $x, y, z \in X$, (1)

$$
v^{x}(\theta, b)-v^{y}(\theta, b)=\lambda\left(u^{x}(\theta, b)-u^{y}(\theta, b)\right)+r^{x y}(\theta)
$$

for all $\theta \in \Theta$ and $b \in B_{\theta}$;

(2) $\sum_{\theta \in \Theta} r^{x y}(\theta)=0$;

(3) $r^{y x}=-r^{x y}$

(4) $r^{x y}+r^{y z}=r^{x z}$.

Define $\beta^{e}=v^{e}$ and $\beta^{x}=r^{x e}+\beta^{e}$ for every $x \neq e$.

\subsection{Implication of Binary Independence}

Lemma 21 For every $\theta \in \Theta$ with $\left|B_{\theta} \backslash\{x, y\}\right| \geqq 2, u^{x y}(\theta, b)=u^{x y}\left(\theta, b^{\prime}\right)$ for all $b, b^{\prime} \in$ $B_{\theta} \backslash\{x, y\}$.

Proof: Without loss, pick any $p^{*} \in P$ such that $u^{x y} \cdot p^{*}=0$. Fix $\theta \in \Theta$ with $\left|B_{\theta} \backslash\{x, y\}\right| \geqq$ 2 , and consider any $\{p(b \mid \theta)\}_{b \in B_{\theta} \backslash\{x, y\}},\{q(b \mid \theta)\}_{b \in B_{\theta} \backslash\{x, y\}}$ that satisfy $\sum_{b \in B_{\theta} \backslash\{x, y\}} p(b \mid \theta)=$ $\sum_{b \in B_{\theta} \backslash\{x, y\}} q(b \mid \theta)=\sum_{b \in B_{\theta} \backslash\{x, y\}} p^{*}(b \mid \theta)$. By condition (i) in the monotonicity axiom, replacing $\left\{p^{*}(b \mid \theta)\right\}_{b \in B_{\theta} \backslash\{x, y\}}$ by $\{p(b \mid \theta)\}_{b \in B_{\theta} \backslash\{x, y\}}$ or $\{q(b \mid \theta)\}_{b \in B_{\theta} \backslash\{x, y\}}$ does not change the indifference condition. Hence we have

$$
\sum_{b \in B_{\theta} \backslash\{x, y\}} u^{x y}(\theta, b)(p(b \mid \theta)-q(b \mid \theta))=0 .
$$

Since $\{p(b \mid \theta)\}_{b \in B_{\theta} \backslash\{x, y\}}$ and $\{q(b \mid \theta)\}_{b \in B_{\theta} \backslash\{x, y\}}$ are arbitrary given the above restriction, we have $u^{x y}(\theta, b)=u^{x y}\left(\theta, b^{\prime}\right)$ for all $b, b^{\prime} \in B_{\theta}$.

Similar arguments deliver the following lemmata.

Lemma $22 u^{x y}(\theta, x)>u^{x y}(\theta, b)$ for all $\theta \in \Theta$ with $x \in B_{\theta}$ and $b \in B_{\theta} \backslash\{x\}$.

Lemma $23 u^{x y}(\theta, b)>u^{x y}(\theta, y)$ for all $\theta \in \Theta$ with $y \in B_{\theta}$ and $b \in B_{\theta} \backslash\{y\}$.

\subsection{Proof of Theorem 8}

Assume that $\Theta_{x} \neq \emptyset$ for all $x \in X$, and $\left|B_{\theta}\right| \geqq 3$ for all $\theta \in \Theta$.

For each $\theta \in \Theta$, fix some $b^{-x y}(\theta) \in B_{\theta} \backslash\{x, y\}$. The above lemma ensures that the choice of $b^{-x y}(\theta)$ does not matter. Define $\widehat{u}^{x y} \in \mathbb{R}^{\sum_{\theta \in \Theta}\left|B_{\theta}\right|}$ by

1. if $x, y \in B_{\theta}$,

$$
\begin{aligned}
& \widehat{u}^{x y}(\theta, x)=u^{x y}(\theta, x)-u^{x y}\left(\theta, b^{-x y}(\theta)\right) \\
& \widehat{u}^{x y}(\theta, y)=u^{x y}(\theta, y)-u^{x y}\left(\theta, b^{-x y}(\theta)\right) \\
& \widehat{u}^{x y}(\theta, b)=0 \quad \text { for all } b \in B_{\theta} \backslash\{x, y\}
\end{aligned}
$$


2. if $x \in B_{\theta}$ and $y \notin B_{\theta}$,

$$
\begin{aligned}
& \widehat{u}^{x y}(\theta, x)=u^{x y}(\theta, x)-u^{x y}\left(\theta, b^{-x y}(\theta)\right) \\
& \widehat{u}^{x y}(\theta, b)=0 \text { for all } b \in B_{\theta} \backslash\{x\}
\end{aligned}
$$

3. if $x \notin B_{\theta}$ and $y \in B_{\theta}$,

$$
\begin{aligned}
& \widehat{u}^{x y}(\theta, y)=u^{x y}(\theta, y)-u^{x y}\left(\theta, b^{-x y}(\theta)\right) \\
& \widehat{u}^{x y}(\theta, b)=0 \text { for all } b \in B_{\theta} \backslash\{y\}
\end{aligned}
$$

4. if $x, y \notin B_{\theta}$,

$$
\widehat{u}^{x y}(\theta, b)=0 \text { for all } b \in B_{\theta}
$$

\section{Lemma 24}

$$
\widehat{u}^{x y} \cdot p=u^{x y} \cdot p
$$

for all $p \in P$.

Proof: Let $\Theta_{x}=\left\{\theta \in \Theta: x \in B_{\theta}\right\}$ and $\Theta_{y}=\left\{\theta \in \Theta: y \in B_{\theta}\right\}$. By Binary Independence, we have

$$
\begin{aligned}
& \sum_{\theta \in \Theta} \sum_{b \in B_{\theta}} u^{x y}(\theta, b) p(b \mid \theta) \\
= & \sum_{\theta \in \Theta_{x} \cap \Theta_{y}}\left(\left(u^{x y}(\theta, x)-u^{x y}\left(\theta, b^{-x y}(\theta)\right) p(x \mid \theta)+\left(u^{x y}(\theta, y)-u^{x y}\left(\theta, b^{-x y}(\theta)\right) p(y \mid \theta)\right)\right)\right. \\
& +\sum_{\theta \in \Theta_{x} \backslash \Theta_{y}}\left(u^{x y}(\theta, x)-u^{x y}\left(\theta, b^{-x y}(\theta)\right) p(x \mid \theta)\right. \\
& +\sum_{\theta \in \Theta_{y} \backslash \Theta_{x}}\left(u^{x y}(\theta, y)-u^{x y}\left(\theta, b^{-x y}(\theta)\right) p(y \mid \theta)\right. \\
& +\sum_{\theta \in \Theta \backslash\left(\Theta_{x} \cup \Theta_{y}\right)} u^{x y}\left(\theta, b^{-x y}(\theta)\right) \\
& +\sum_{\theta \in \Theta_{x} \cap \Theta_{y}} u^{x y}\left(\theta, b^{-x y}(\theta)\right) \\
& +\sum_{\theta \in \Theta_{x} \backslash \Theta_{y}} u^{x y}\left(\theta, b^{-x y}(\theta)\right) \\
& +\sum_{\theta \in \Theta_{y} \backslash \Theta_{x}} u^{x y}\left(\theta, b^{-x y}(\theta)\right)
\end{aligned}
$$

By Dummy, the terms from 4th to 7th on the right-hand-side add up to zero. Therefore the right-hand-side is equal to $\widehat{u}^{x y} \cdot p$. 
By construction, we have

$$
\widehat{u}^{y x}=-\widehat{u}^{x y}
$$

for all $x, y \in X$. Also, the above lemma guarantees that

$$
\widehat{u}^{x y} \cdot p+\widehat{u}^{y z} \cdot p=\widehat{u}^{x z} \cdot p
$$

for all $p \in P$.

Lemma 25 For all $x, y, z \in X$ and $\theta \in \Theta_{x}$,

$$
\widehat{u}^{x y}(\theta, x)=\widehat{u}^{x z}(\theta, x)
$$

Proof: Let $\Theta_{\{x, y, z\}}=\left\{\theta \in \Theta: B_{\theta}=\{x, y, z\}\right\}$. Note that

$$
\begin{aligned}
= & \sum_{\theta \in \Theta_{\{x, y, z\}}}\left(\widehat{u}^{x y} \cdot p(\theta, x) p(x \mid \theta)+\widehat{u}^{x y}(\theta, y) p(y \mid \theta)+\widehat{u}^{x y}(\theta, z)\right) \\
& +\sum_{\theta \in \Theta_{x} \backslash \Theta_{\{x, y, z\}}} \widehat{u}^{x y}(\theta, x) p(x \mid \theta)+\sum_{\theta \in \Theta_{y} \backslash \Theta_{\{x, y, z\}}} \widehat{u}^{x y}(\theta, y) p(y \mid \theta), \\
= & \widehat{u}_{\theta \in \Theta_{\{x, y, z\}}}^{y z}\left(\widehat{u}^{y z}(\theta, x) p(x \mid \theta)+\widehat{u}^{y z}(\theta, y) p(y \mid \theta)+\widehat{u}^{y z}(\theta, z)\right) \\
& +\sum_{\theta \in \Theta_{y} \backslash \Theta_{\{x, y, z\}}} \widehat{u}^{y z}(\theta, y) p(y \mid \theta)+\sum_{\theta \in \Theta_{z} \backslash \Theta_{\{x, y, z\}}} \widehat{u}^{y z}(\theta, z) p(z \mid \theta)
\end{aligned}
$$

and

$$
\begin{aligned}
= & \sum_{\theta \in \Theta_{\{x, y, z\}}}^{\widehat{u}^{x z} \cdot p}\left(\widehat{u}^{x z}(\theta, x) p(x \mid \theta)+\widehat{u}^{x z}(\theta, y) p(y \mid \theta)+\widehat{u}^{x z}(\theta, z)\right) \\
& +\sum_{\theta \in \Theta_{x} \backslash \Theta_{\{x, y, z\}}} \widehat{u}^{x z}(\theta, x) p(x \mid \theta)+\sum_{\theta \in \Theta_{z} \backslash \Theta_{\{x, y, z\}}} \widehat{u}^{x z}(\theta, z) p(z \mid \theta) .
\end{aligned}
$$

Case 1: For $\theta \in \Theta_{x} \backslash \Theta_{\{x, y, z\}}$, since one may vary $p(x \mid \theta)$ between 0 and 1 freely, we have

$$
\widehat{u}^{x y}(\theta, x)=\widehat{u}^{x z}(\theta, x) .
$$

Case 2: For $\theta \in \Theta_{\{x, y, z\}}$, since one may vary $p(x \mid \theta)$ linearly independently of other numbers, we have

$$
\widehat{u}^{x y}(\theta, x)+\widehat{u}^{y z}(\theta, x)=\widehat{u}^{x z}(\theta, x) .
$$

On the other hand, by construction $\widehat{u}^{y z}(\theta, x)=0$, hence

$$
\widehat{u}^{x y}(\theta, x)=\widehat{u}^{x z}(\theta, x) \text {. }
$$


Now, define $u^{x} \in \mathbb{R}^{\left|\Theta_{x}\right|}$ by

$$
u^{x}(\theta)=\widehat{u}^{x e}(\theta, x)
$$

for each $\theta \in \Theta_{x}$. Then, we have

$$
\begin{aligned}
& \sum_{\theta \in \Theta_{x}} u^{x}(\theta) p(x \mid \theta)-\sum_{\theta \in \Theta_{y}} u^{y}(\theta) p(y \mid \theta) \geqq 0 \\
& \Longleftrightarrow \sum_{\theta \in \Theta_{x}} u^{x}(\theta) p(x \mid \theta)-\sum_{\theta \in \Theta_{e}} u^{e}(\theta) p(e \mid \theta)-\left(\sum_{\theta \in \Theta_{y}} u^{y}(\theta) p(y \mid \theta)-\sum_{\theta \in \Theta_{e}} u^{e}(\theta) p(e \mid \theta)\right) \geqq 0 \\
& \Longleftrightarrow \widehat{u}^{x e} \cdot p-\widehat{u}^{y e} \cdot p \geqq 0 \\
& \Longleftrightarrow \widehat{u}^{x e} \cdot p+\widehat{u}^{e y} \cdot p \geqq 0 \\
& \Longleftrightarrow \widehat{u}^{x y} \cdot p \geqq 0 \\
& \Longleftrightarrow u^{x y} \cdot p \geqq 0 \\
& \Longleftrightarrow \succsim_{p} y .
\end{aligned}
$$

Uniqueness: Recall the uniqueness result in Lemma 13 that if there is another series of vectors $\left\{v^{x y}\right\}_{x \in X}$ satisfies the above condition, then there is a scaler $\lambda$ and a series of vectors in $\mathbb{R}^{|\Theta|}$, denoted $\left\{r^{x y}\right\}_{x, y \in X, x \neq y}$, such that for every $x, y, z \in X$,

$$
v^{x y}(\theta, b)=\lambda u^{x y}(\theta, b)+r^{x y}(\theta)
$$

for all $\theta \in \Theta$ and $b \in B_{\theta}$;

(2) $\sum_{\theta \in \Theta} r^{x y}(\theta)=0$

(3) $r^{y x}=-r^{x y}$

(4) $r^{x y}+r^{y z}=r^{x z}$.

Let $\left\{\widehat{v}^{x y}\right\}_{x \in X}$ be the series of vectors obtained from $\left\{v^{x y}\right\}_{x \in X}$ as in the current argument. Since all the terms in $r^{x y}$ are canceled out along the above procedure, we have $\widehat{u}^{x y}=\lambda \widehat{v}^{x y}$ for all $x, y \in X$.

\subsection{Proof of Theorem 10}

Lemma 26 For all $x, y, z \in X$ and $\theta \in \Theta$ with $B_{\theta}=\{x, y\}$,

$$
\frac{u^{x y}(\theta, x)-u^{x y}(\theta, y)}{2}=u^{x z}(\theta, x)-u^{x z}(\theta, y) \text {. }
$$

Proof: Given $\theta \in \Theta$ with $B_{\theta}=\{x, y\}$, let $q_{\theta}$ be the distribution that is degenerated on $x$. Let $p \in P$ be such that

$$
u^{y z} \cdot p=0, \quad u^{x y} \cdot p<0, \quad u^{x z} \cdot p<0 .
$$


Notice that $u^{x z} \cdot p=u^{x y} \cdot p+u^{y z} \cdot p=u^{x y} \cdot p$. Thus, let $u^{x y} \cdot p=u^{x z} \cdot p=-c$, where $c>0$ is a sufficiently small number.

- By Diversity and Continuity, one can find $p^{1}, p^{2} \in \operatorname{relint} P$ such that $y \succ_{p^{1}} z \succ_{p^{1}} x$ and $z \succ_{p^{2}} y \succ_{p^{2}} x$, where $\left|u^{x y} \cdot p^{1}\right|,\left|u^{x z} \cdot p^{1}\right|,\left|u^{x y} \cdot p^{2}\right|,\left|u^{x z} \cdot p^{2}\right|$ are sufficiently small. By Combination, one can find $\mu$ so that $p=(1-\mu) p^{1}+\mu p^{2} \in$ relint $P$ satisfies $y \sim_{p} z \succ_{p} x$, where $\left|u^{x z} \cdot p\right|$ is sufficiently small.

Let $\lambda \in(0,1)$ be such that

$$
\left(u^{x y}(\theta, x)-u^{x y}(\theta, y)\right)(1-p(x \mid \theta)) \lambda=c,
$$

where $x \sim_{(1-\lambda) p+\lambda\left(q_{\theta}, p_{-\theta}\right)} y$ is the case. By Proportionality, we have $x \sim_{\left(1-\frac{\lambda}{2}\right) p+\frac{\lambda}{2}\left(q_{\theta}, p_{-\theta}\right)} z$, in which

$$
\left(u^{x y}(\theta, x)-u^{x y}(\theta, y)\right)(1-p(x \mid \theta)) \frac{\lambda}{2}=c
$$

holds. Thus we obtain the desired result.

For each $\theta \in \Theta$ with $B_{\theta} \neq\{x, y\}$, fix some $b^{-x y}(\theta) \in B_{\theta} \backslash\{x, y\}$. The above lemma ensures that the choice of $b^{-x y}(\theta)$ does not matter.

Define $\widehat{u}^{x y} \in \mathbb{R}^{\sum_{\theta \in \Theta}\left|B_{\theta}\right|}$ by

1. if $B_{\theta}=\{x, y\}$,

$$
\widehat{u}^{x y}(\theta, x)=\left(u^{x y}(\theta, x)-u^{x y}(\theta, y)\right) / 2, \quad \widehat{u}^{x y}(\theta, y)=\left(u^{x y}(\theta, y)-u^{x y}(\theta, x)\right) / 2
$$

2. otherwise, same as before

\section{Lemma 27}

$$
\widehat{u}^{x y} \cdot p=u^{x y} \cdot p
$$

for all $p \in P$.

Proof: Let $\Theta_{\{x, y\}}=\left\{\theta \in \Theta: B_{\theta}=\{x, y\}\right\}, \Theta_{x}=\left\{\theta \in \Theta: x \in B_{\theta}\right\}$ and $\Theta_{y}=\{\theta \in \Theta$ : 
$\left.y \in B_{\theta}\right\}$. By Binary Independence, we have

$$
\begin{aligned}
& \sum_{\theta \in \Theta} \sum_{b \in B_{\theta}} u^{x y}(\theta, b) p(b \mid \theta) \\
= & \sum_{\theta \in \Theta_{\{x, y\}}}\left(\frac{u^{x y}(\theta, x)-u^{x y}(\theta, y)}{2} p(x \mid \theta)+\frac{u^{x y}(\theta, y)-u^{x y}(\theta, x)}{2} p(y \mid \theta)\right) \\
& +\sum_{\theta \in \Theta_{x} \cap \Theta_{y} \backslash \Theta_{\{x, y\}}}\left(\left(u^{x y}(\theta, x)-u^{x y}\left(\theta, b^{-x y}(\theta)\right) p(x \mid \theta)+\left(u^{x y}(\theta, y)-u^{x y}\left(\theta, b^{-x y}(\theta)\right) p(y \mid \theta)\right)\right)\right. \\
& +\sum_{\theta \in \Theta_{x} \backslash \Theta_{y}}\left(u^{x y}(\theta, x)-u^{x y}\left(\theta, b^{-x y}(\theta)\right) p(x \mid \theta)\right. \\
& +\sum_{\theta \in \Theta_{y} \backslash \Theta_{x}}\left(u^{x y}(\theta, y)-u^{x y}\left(\theta, b^{-x y}(\theta)\right) p(y \mid \theta)\right. \\
& +\sum_{\theta \in \Theta \backslash\left(\Theta_{x} \cup \Theta_{y}\right)} u^{x y}\left(\theta, b^{-x y}(\theta)\right) \\
& +\sum_{\theta \in \Theta_{\{x, y\}}} \frac{u^{x y}(\theta, x)+u^{x y}(\theta, y)}{2} \\
& +\sum_{\theta \in \Theta_{x} \cap \Theta_{y} \backslash \Theta_{\{x, y\}}} u^{x y}\left(\theta, b^{-x y}(\theta)\right) \\
& +\sum_{\theta \in \Theta_{x} \backslash \Theta_{y}} u^{x y}\left(\theta, b^{-x y}(\theta)\right) \\
+ & \sum_{\theta \in \Theta_{y} \backslash \Theta_{x}} u^{x y}\left(\theta, b^{-x y}(\theta)\right)
\end{aligned}
$$

By Strong Dummy, the terms from fifth to ninth on the right-hand-side add up to zero. Therefore the right-hand-side is equal to $\widehat{u}^{x y} \cdot p$.

By construction, we have

$$
\widehat{u}^{y x}=-\widehat{u}^{x y}
$$

for all $x, y \in X$. Also, the above lemma guarantees that

$$
\widehat{u}^{x y} \cdot p+\widehat{u}^{y z} \cdot p=\widehat{u}^{x z} \cdot p
$$

for all $p \in P$.

Lemma 28 For all $x, y, z \in X$ and $\theta \in \Theta_{x}$,

$$
\widehat{u}^{x y}(\theta, x)=\widehat{u}^{x z}(\theta, x)
$$

Proof: When $\theta$ is such that $B_{\theta}=\{x, y\}$, it follows from

$$
\widehat{u}^{x y}(\theta, x)=\frac{u^{x y}(\theta, x)-u^{x y}(\theta, y)}{2}=u^{x z}(\theta, x)-u^{x z}(\theta, y)=\widehat{u}^{x z}(\theta, x) .
$$

For the other cases, it follows from the same argument as before. 
Now, define $u^{x} \in \mathbb{R}^{\left|\Theta_{x}\right|}$ by

$$
u^{x}(\theta)=\widehat{u}^{x e}(\theta, x)
$$

for each $\theta \in \Theta_{x}$. Then, we have

$$
\begin{aligned}
& \sum_{\theta \in \Theta_{x}} u^{x}(\theta) p(x \mid \theta)-\sum_{\theta \in \Theta_{y}} u^{y}(\theta) p(y \mid \theta) \geqq 0 \\
& \Longleftrightarrow \sum_{\theta \in \Theta_{x}} u^{x}(\theta) p(x \mid \theta)-\sum_{\theta \in \Theta_{e}} u^{e}(\theta) p(e \mid \theta)-\left(\sum_{\theta \in \Theta_{y}} u^{y}(\theta) p(y \mid \theta)-\sum_{\theta \in \Theta_{e}} u^{e}(\theta) p(e \mid \theta)\right) \geqq 0 \\
& \Longleftrightarrow \widehat{u}^{x e} \cdot p-\widehat{u}^{y e} \cdot p \geqq 0 \\
& \Longleftrightarrow \widehat{u}^{x e} \cdot p+\widehat{u}^{e y} \cdot p \geqq 0 \\
& \Longleftrightarrow \widehat{u}^{x y} \cdot p \geqq 0 \\
& \Longleftrightarrow u^{x y} \cdot p \geqq 0 \\
& \Longleftrightarrow x{ }^{x} y .
\end{aligned}
$$

Uniqueness: Recall the uniqueness result in Lemma 13 that if there is another series of vectors $\left\{v^{x y}\right\}_{x \in X}$ satisfies the above condition, then there is a scaler $\lambda$ and a a series of vectors in $\mathbb{R}^{|\Theta|}$, denoted $\left\{r^{x y}\right\}_{x, y \in X, x \neq y}$, such that for every $x, y, z \in X$,

$$
v^{x y}(\theta, b)=\lambda u^{x y}(\theta, b)+r^{x y}(\theta)
$$

for all $\theta \in \Theta$ and $b \in B_{\theta}$;

(2) $\sum_{\theta \in \Theta} r^{x y}(\theta)=0$;

(3) $r^{y x}=-r^{x y}$

(4) $r^{x y}+r^{y z}=r^{x z}$.

Let $\left\{\widehat{v}^{x y}\right\}_{x \in X}$ be the series of vectors obtained from $\left\{v^{x y}\right\}_{x \in X}$ as in the current argument. Since all the terms in $r^{x y}$ are canceled out along the above procedure, we have $\widehat{u}^{x y}=\lambda \widehat{v}^{x y}$ for all $x, y \in X$.

\subsection{Proof of Corollary 1}

Lemma 29 For all $x, y \in X$ and $B \in \Theta$ with $x, y \in B, u^{x}(B)=u^{y}(B)$.

Proof: Let $p \in P$ be such that $p(x \mid B)=p(y \mid B)=\frac{1}{2}$, and $x, y$ are never chosen elsewhere and the distribution there is uniform over the other alternatives.

By applying Neutrality with regard to the permutation between $x$ and $y$, we have $x \sim_{p} y$, which implies $\frac{1}{2} u^{x}(B)=\frac{1}{2} u^{y}(B)$. Hence $u^{x}(B)=u^{y}(B)$.

Thus, there exists a function $u: \mathcal{B} \rightarrow \mathbb{R}_{++}$such that

$$
u^{x}(B)=u(B)
$$

for all $B \in \mathcal{B}$ with $x \in B$. The remaining is to show that $u(B)$ depends only on the cardinality of $B$. 
Lemma 30 For all $B, C \in \mathcal{B}$ with $|B|=|C|, u(B)=u\left(B^{\prime}\right)$.

Proof: Pick $x \in B \backslash C$ and $y \in C \backslash B$. Let $p \in P$ be such that

(i) $p(x \mid B)=1, p(y \mid C)=1, p(x \mid\{x, y\})=p(y \mid\{x, y\})=\frac{1}{2}$, and $x, y$ are never chosen elsewhere and the distribution there is uniform over the other alternatives. By applying Neutrality with regard to the permutation between $x$ and $y$, we have $x \sim_{p} y$, which implies $u(B)+\frac{1}{2} u(\{x, y\})=u(C)+\frac{1}{2} u(\{x, y\})$. Hence $u(B)=u(C)$.

\section{References}

[1] Arrow, Kenneth., Rational Choice Functions and Orderings, Econometrica 26 (1959) $121-7$.

[2] Bermheim, Douglas and Antonio Rangel, Toward Choice-Theoretic Foundations for Behavioral Welfare Economics, American Economic Review Papers and Proceedings, 97(2), May 2007, 464-470.

[3] Bermheim, Douglas and Antonio Rangel, Beyond Revealed Preference: Choice Theoretic Foundations for Behavioral Welfare Economics, working paper, October 2007.

[4] Camerer, C., D. Loewenstein, and D. Prelec, Neuroeconomics: How Neuroscience can Inform Economics, Journal of Economic Literature 43, 9-64, 2005.

[5] Carmichael, H. Lorne and W Bentley MacLeod, How should a Behavioral Economist do Welfare Economics?, working paper, Queen's University, 2002.

[6] Chernoff, H., Rational Selection of Decision Functions, Econometrica 22, 422-43, 1954.

[7] Falmagne, J. C., A Representation Theorem for Finite Random Scale Systems, Journal of Mathematical Psychology, 18(1978), 52-72.

[8] Florenzano, M. and C. Le Van, Finite Dimensional Convexity and Optimization, Springer, 2001.

[9] Green, Jerry R. and Daniel A. Hojman, Choice, Rationality, and Welfare Measurement, working paper, Harvard University, 2007.

[10] Gilboa, Itzhak, and David Schmeidler, Case-Based Decision Theory, Quarterly Journal of Economics, 110 (1995) 605-639.

[11] Gilboa, Itzhak, and David Schmeidler, Act similarity in case-based decision theory, Economic Theory, Vol. 9 (1997) 47-61.

[12] Gilboa, Itzhak, and David Schmeidler, Inductive Inference: An Axiomatic Approach, Econometrica, 71 (2003), 1-26. 
[13] Gilboa, Itzhak, and David Schmeidler, A derivation of expected utility maximization in the context of a game, Games and Economic Behavior, Volume 44, Issue 1, July 2003, Pages 172-182.

[14] Kahneman, D., Slovic, P., and Tversky, A. Judgment under uncertainty: Heuristics and biases, New York, Cambridge University Press, 1982.

[15] Kahneman, D., and Tversky, A. (Eds.), Choices, values and frames, New York, Cambridge University Press, 2000.

[16] Kahneman, Daniel, Peter P. Wakker and Rakesh Sarin, Back to Bentham? Exploration of Experienced Utility, Quarterly Journal of Economics, Volume 112, Issue 2, 1997, Pages 375-405.

[17] Kalai, Gil, Ariel Rubinstein and Rani Spiegler, Rationalizing Choice Functions by Mutltiple Rationales, Econometrica, 70 (2002), 2481-2488.

[18] Karni, Edi, David Schmeidler and Karl Vind, On State Dependent Preferences and Subjective Probabilities, Econometrica Vol. 51, No. 4 (July 1983), 1021-1031.

[19] Kreps, David, Notes on the Theory of Choice, Westview Press, 1988.

[20] Luce, R. Duncan, A Probabilistic Theory of Utility, Econometrica, Vol. 26, No. 2. (Apr., 1958), pp. 193-224.

[21] Mangasarian, O.L., Nonlinear programming, McGraw-Hill, New York, 1969.

[22] Mitchell, Gregory, Libertarian Paternalism Is an Oxymoron, Northwestern University Law Review, Vol. 99, No. 3, 2005.

[23] Masatlioglu, Yusufcan and Efe Ok, Rational choice with status quo bias, Journal of Economic Theory, 121 (2005), No. 1, 1-29.

[24] Rubinstein, Ariel and Yuval Salant, A Model of Choice from Lists, Theoretical Economics, 1 (2006), No. 1, 3-17.

[25] Rubinstein, Ariel and Yuval Salant, (A,f): Choice with Frames, to appear in Review of Economic Studies, 2007.

[26] Samuelson, Paul A., A note on the pure theory of consumer behavior, Economica, 5(17): 61-71, 1938.

[27] Samuelson, Paul A., Consumption theory in terms of revealed preference. Economica, 15(60):243-253, 1948.

[28] Sen, A.K., Choice Functions and Revealed Preferences, Review of Economic Studies 38, 307-17, 1971.

[29] Thaler, Richard H., The Winner's Curse: Paradoxes and Anomalies of Economic Life, Free Press, 1991. 
[30] Thaler, Richard H. and Cass Sunstein, Libertarian Paternalism is Not an Oxymoron, University of Chicago Law Review 70 (4), (2003): 1159-1202.

[31] Thaler, Richard H. and Cass Sunstein, Libertarian Paternalism, American Economic Review 93 (2), (2003): 175-179.

[32] Tyson, Christopher J., Cognitive constraints, contraction consistency, and the satisficing criterion, Journal of Economic Theory, Volume 138, Issue 1, January 2008, Pages 51-70.

[33] Young, Peyton, Social Choice Scoring Functions, SIAM Journal on Applied Mathematics, 28 (1975), 824-838. 